Summary of US-Japan Exchange 2004 New Directions and Physics for Compact Toroids

T. Intrator, M. Nagata, A. Hoffman, H. Guo, L. Steinhauer, D. Ryutov, R. Miller, S. Okada

August 17, 2005

Journal of Fusion Energy 
This document was prepared as an account of work sponsored by an agency of the United States Government. Neither the United States Government nor the University of California nor any of their employees, makes any warranty, express or implied, or assumes any legal liability or responsibility for the accuracy, completeness, or usefulness of any information, apparatus, product, or process disclosed, or represents that its use would not infringe privately owned rights. Reference herein to any specific commercial product, process, or service by trade name, trademark, manufacturer, or otherwise, does not necessarily constitute or imply its endorsement, recommendation, or favoring by the United States Government or the University of California. The views and opinions of authors expressed herein do not necessarily state or reflect those of the United States Government or the University of California, and shall not be used for advertising or product endorsement purposes. 


\section{Summary of US-Japan exchange 2004 New directions and physics for compact toroids}

T.Intrator (LANL), M. Nagata (Univ Hyogo), A. Hoffman, H. Guo, L. Steinhauer (Univ Washington, RPPL), D. Ryutov (LLNL), R. Miller (Decysive Systems, USA), S. Okada (Osaka Univ.)

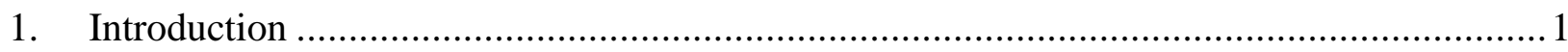

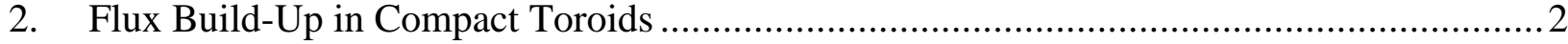

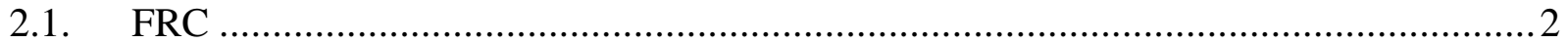

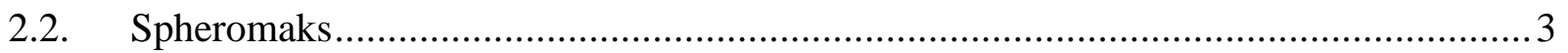

2.3. Inductive Flux Build-Up..................................................................................

3. General relaxation to a finite plasma pressure plasma........................................................ 3

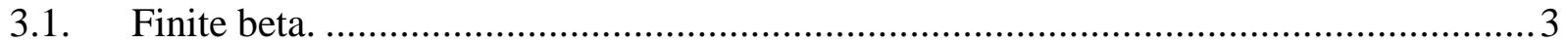

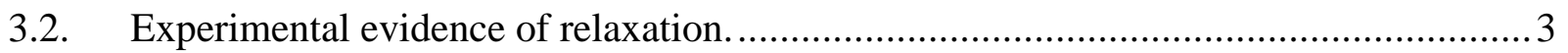

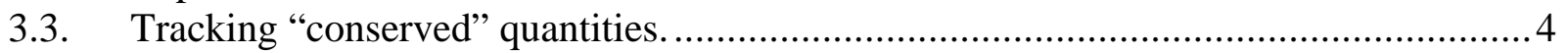

3.4. Self-organization in helicity-driven systems ........................................................ 4

3.5. Jets, collimation, mass ingestion intrinsic to spheromak formation ............................. 4

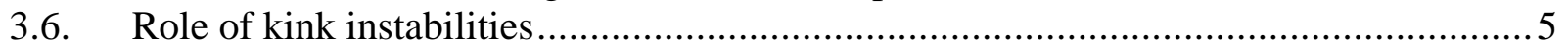

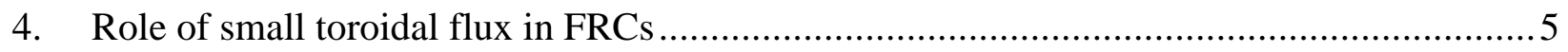

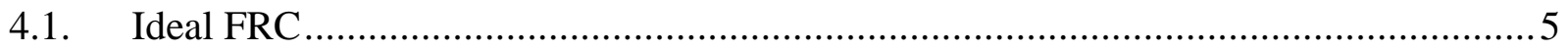

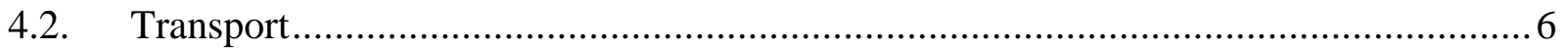

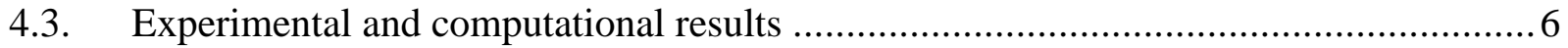

4.4. Existence of intermediate states between FRC and spheromak.................................. 7

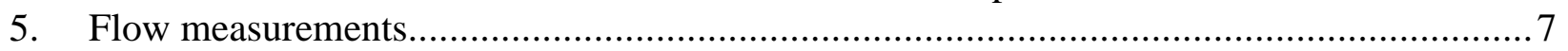

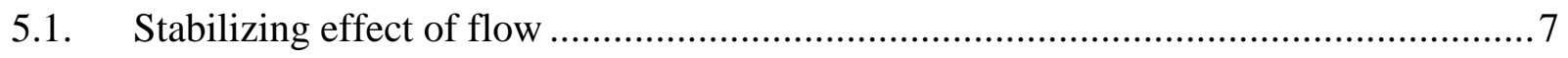

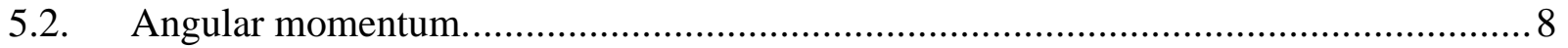

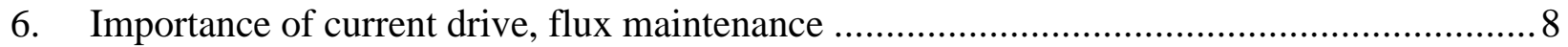

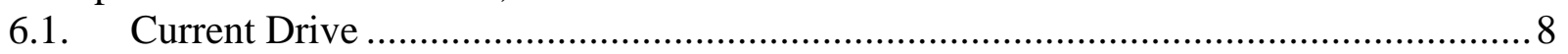

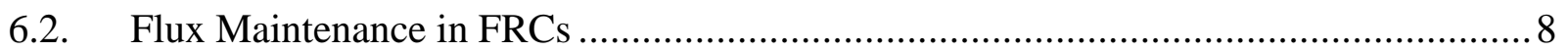

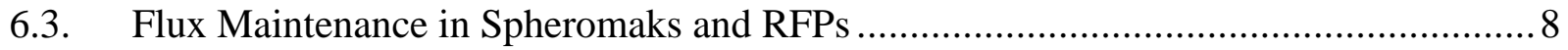

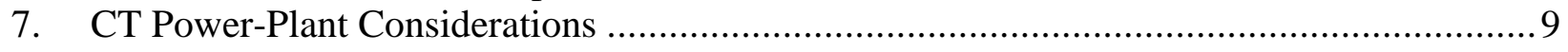

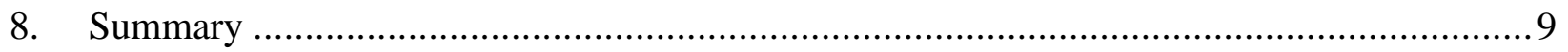

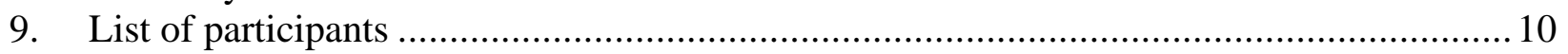

\section{Introduction}

This exchange workshop was an open meeting coordinated by the P-24 Plasma Physics Group at Los Alamos National Laboratory. We brought together scientists from institutions in the US and Japan who are researching the various and complementary types of Compact Toroids (CT). Many concepts, including both experimental and theoretical investigations, are represented. The range spans Field Reversed Configuration (FRC), spheromak, Reversed Field Pinch (RFP), spherical tokamaks, linear devices dedicated to fundamental physics studies, and hybrid 
transitions that bridge multiple configurations. The participants represent facilities on which significant experiments are now underway: FRC Injection experiment (FIX), Translation Confinement experiment (TCS), Nihon-University Compact Torus Experiment (NUCTE), HITSI (Helicity Injection experiment, Steady Inductive Helicity Injection (HIT-SIHI)), Field Reversed Configuration experiment -Liner (FRX-L), TS-3/4, Sustained Spheromak Experiment (SSPX), Relaxation Scaling Experiment (RSX), HIST, Caltech Spheromak, or in the design process such as MRX-FRC (PPPL), Pulsed High Density experiment (PHD at UW).

Several new directions and results in compact toroid (CT) research have recently emerged, including neutral-beam injection, rotating magnetic fields, flux build up from Ohmic boost coils, electrostatic helicity injection techniques, CT injection into other large devices, and high density configurations for applications to magnetized target fusion and translational compression of CT's. CT experimental programs in both the US and Japan have also shown substantial progress in the control and sustainment of CT's. Both in theory and experiment, there is increased emphasis on 3D dynamics, which is also related to astrophysical and space physics issues. 3D data visualization is now frequently used for experimental data display. There was much discussion of the effects of weak toroidal fields in FRC's and possible implications for transport and relaxation.

A variety of key themes surrounding the physics of CT's were found to recur during this conference. These included questions and answers touching upon magnetic flux build up in CT's, generalized relaxation processes that extend beyond the Taylor picture, the importance of plasma flows, toroidal magnetic fields in FRC's, and CT power plant considerations. This document briefly outlines the tenor of these discussions.

\section{Flux Build-Up in Compact Toroids}

A key technological challenge in compact toroid research is providing enough flux to reach more reactor relevant parameters (i.e., large ratio of scale length to ion gyro-radius). In some cases, it is even a problem to provide enough flux to efficiently utilize neutral beams for current drive or stabilization experiments. The methods of forming high beta FRCs and low beta spheromaks are different due to their different magnetic field topologies, but the scale of each is governed by its poloidal flux. An attractive method of increasing the flux, discussed at this workshop, is the temporary use of a central flux core, and then translating the CT off the flux core so that it would regain the desirable properties of a singly connected object.

\subsection{FRC}

In FRCs the plasma pressure must balance the poloidal field forces. If purely Ohmic processes are relied on to heat the plasma, the heating scales as $\mathrm{P} \Omega \sim \eta \mathrm{j}^{2} \sim \eta(\mathrm{B} / \mathrm{r})^{2}$. Radiated power scales as $\mathrm{n}^{2} \sim\left(\mathrm{B}^{2} / \mathrm{T}\right)^{2}$ so that as $\mathrm{B}$ is increased it is easy to encounter radiative collapse unless the temperature also rises rapidly. Rapid, non-Ohmic heating occurs in fast theta-pinches, which is why high temperatures, high densities, and high beta are easily achievable by that method, but the technology becomes difficult in scaling above the $10 \mathrm{mWb}$ flux level. Combining opposite helicity spheromaks, where the merging occurs rapidly, is a recent method of forming FRCs, but it remains to be seen how high a poloidal flux can be obtained in this manner. Rotating Magnetic Fields (RMF) can also be used to form FRCs at low densities and, if the impurity level can be kept low, it should be possible to have the Ohmic heating surpass radiative losses, allowing higher temperatures and magnetic fields than in present devices. 
FRCs are also candidates for pulsed, high density fusion. The amount of flux required for favorable energy balance will scale inversely with B to some power between 1 and 2 (depending on lifetime scaling). If theta-pinch formation is used and operation at non-destructive magnetic field pressures is desired, it will be essential to maximize flux trapping during the theta pinch formation and flux retention during the subsequent compression process.

\subsection{Spheromaks}

Spheromaks can be formed in a similar manner to FRC's using flux cores. Since the configuration is nearly force free, the densities will be lower and the formation timescales can be longer with reduced requirements for externally applied power. It is not known at what size this will become impractical. Most spheromaks are formed using coaxial guns with a given initial poloidal flux. However, by continually injecting toroidal flux and helicity, the poloidal flux can be increased through flux conversion. This process involves electrodes and significant 3-D turbulent processes, so it may be difficult to build the flux up beyond a certain level even though the plasma density may be kept low. A unique, electrode-less inductive helicity scheme is currently being developed.

\subsection{Inductive Flux Build-Up}

While theta-pinch formation methods supply GW of power, most spheromak formation processes involve $\sim 100 \mathrm{MW}$, and RMF supplies MW level auxiliary power. It is thus difficult to imagine non-Ohmic methods, such as radio frequency waves or neutral beam injection, being powerful enough to add much to the existing heating or flux build-up. An Ohmic method being proposed is the use of an inner coaxial flux core to add flux. The previous concerns about radiation rates exceeding Ohmic heating will apply, but if the initial density can be kept low it should be possible to overcome low $\mathrm{Z}$ radiation barriers, similar to what is done for standard tokamak startup. RMF produces low density FRCs, and experiments will soon be underway to minimize impurities. Internal flux cores have also been proposed for use in spheromak merging experiments, either to increase the flux of the initial spheromaks or to be applied to the resultant FRC.

\section{General relaxation to a finite plasma pressure plasma}

\subsection{Finite beta.}

Taylor relaxation to a minimum-energy state is a well-known principle that applies, in part, to certain classes of plasmas. However, it predicts force-free states, whereas even the configurations to which the Taylor theory applies best, spheromaks and RFPs, have finite $\beta$ and pressure gradients. A more general relaxation theory is based on the two-fluid model (rather than Taylor's single fluid) and has two helicity invariants instead of one. Analyses of these states show that (1) finite $\beta$ appears in states with significant flow, and (2) the Taylor states are a subclass of the more general states.

\subsection{Experimental evidence of relaxation.}

Results from TCS[Guo2004] and FIX indicate that the poloidal flux of the plasmoid increases significantly in the course of its injection into the confinement chamber. Cowling's theorem [Cowling] states that such a transition requires three-dimensional dynamics, such as in a relaxation. Moreover, the electron helicity is roughly conserved despite the extreme violence of the plasmoid ejection and stopping. Velocity measurements to date are too incomplete to 
determine if the ion helicity is conserved. Axial magnetic compression of the compact toroid would increases the toroidal field if poloidal flux were conserved, but increased toroidal field is not observed in FIX. This may reflect the conversion of toroidal to poloidal flux.

1. H.Y. Guo, A.L. Hoffman, K.E. Miller, L.C. Steinhauer. Phys. Rev. Lett., 92, 245001-1 (2004)

2. T.G. Cowling, Mon. N. Roy. Astron. Soc. 94, 34 (1939).

\subsection{Tracking conserved quantities.}

The "electron" (or magnetic) helicity can be inferred from magnetic probe measurements, as routinely done in spheromaks and lately in low-density FRCs. However, tracking the ion helicity also requires measurements of the velocity fields. Successful measurements of the toroidal flow pattern have exploited the Doppler shift of impurity lines. However, poloidal flow measurements have not been made in compact toroids. Such more complete measurements are essential in the future to determine if the relaxation is regulated by both helicities. Such measurements will also clarify the three-dimensional transitional mechanism (e.g., internal kink modes) by which the initial state relaxes to the final state.

\subsection{Self-organization in helicity-driven systems}

A new research area of Taylor relaxation has been discovered in helicity-driven toroidal systems. The helicity-driven relaxation theory [Taylor1989], extending beyond closed systems, predicts various kinds of driven-relaxed states such as spherical torus (ST) plasmas, spheromaks with a central rod, low-aspect-ratio RFPs, and tokamaks. The experimental finding on HIST [Nagata2003] provides, for the first time, evidence for the existence of the flipped ST state predicted theoretically for negative polarity of the external toroidal field and from the conservation of the sign of magnetic helicity. Self-organization during the transition from normal to flipped relaxed states is closely related to magnetic reconnection of the helically kinked open field lines. New approaches to achieving finite plasma pressure plasmas are now being investigated in helicity-driven systems. Equilibrium calculations based on a two-fluid model show that there is a diamagnetic low q ST with higher beta than a conventional ST. Important future experimental work will drive fast ion flow (e.g., CT injection) for plasma formation.

\subsection{Jets, collimation, mass ingestion intrinsic to spheromak formation}

Experiments on the planar coaxial spheromak source at Caltech show that MHD forces ingest plasma (i.e., mass) from the gas puff valves. The ingested mass convects frozen-in toroidal flux linking the poloidal flux established by the bias coil. Thus mass ingestion is directly associated with helicity ingestion. Because of stagnation of the ingested mass flow, there is a concommitant pile-up of the frozen-in toroidal flux associated with the flow. This toroidal flux concentration results in increased toroidal field and hence increased pinch effect which collimates the current channel (poloidal current) so that a long narrow jet of dense hot plasma is formed. When this jet becomes sufficiently long it satisfies $q=1$ and becomes kink unstable. The nonlinear kink converts toroidal flux into poloidal flux and leads to creation of a spheromak.

1. S. C. Hsu and P. M. Bellan, Experimental Identification of the Kink Instability as a Poloidal Flux Amplification Mechanism for Coaxial Gun Spheromak Formation, Phys Rev. Letters 90, article 215002 (2003)

2. P. M. Bellan, Why current-carrying magnetic flux tubes gobble up plasma and become thin as a result, Physics of Plasmas 10, Pt 2, 1999 (2003) 
3. S. C. Hsu and P. M. Bellan, A laboratory plasma experiment for studying magnetic dynamics of accretion disks and jets, Mon. Not. Royal Astron. Soc, 334, 257 (2002)

4. S. C. Hsu and P. M. Bellan, Study of Magnetic Helicity Injection via Plasma Imaging Using a High-Speed Digital Camera, IEEE Trans Plasma Sciences, 30, 10 (2002)

\subsection{Role of kink instabilities}

The most successful theoretical approach to relaxation of a turbulent magnetohydrodynamic (MHD) system is the Taylor hypothesis [Taylor1974] [Taylor1986] that magnetic energy is minimized subject to the constraint of constant global magnetic helicity. Since this is essentially a thermodynamic argument, the actual 3D dynamics underlying the relaxation process remain unexplained. For example, in coaxial gun spheromaks a dynamo mechanism can convert injected toroidal flux into required poloidal flux. Cowling's theorem [Cowling1939] requires non axisymmetric processes to accomplish this. In many situations in the laboratory and in nature, kink-like instabilities are observed to deform an axisymmetric plasma configuration into a 3D one [Hsu2003]. Examples of this were also shown in RSX [Furno2003], in which helical deformations soon dominate the evolution of initially straight current channels.

There was a general appreciation that relaxation is a common effect for many of the CT concepts, and that the development of kink instabilities seems to be a common thread in many situations. Many Innovative Confinement Concept approaches contain large plasma beta and pressure gradients and therefore are far from force free and clearly are not relaxed in the Taylor sense. One possible scenario for pulsed or cyclic CT operation might be to charge the configuration with magnetic flux and subsequently wait while it relaxes and dissipates this flux, and then repeat the cycle.

1. J.B. Taylor and M.F. Turner, Nucl. Fusion 29, 219 (1989)

2. M. Nagata et al. Phys. Rev. Lett. 90, 225001 (2003).

3. T.G. Cowling, Mon. N. Roy. Astron. Soc. 94, 34 (1939).

4. J.B. Taylor, Relaxation of toroidal plasma and generation of reverse magnetic fields, Phys. Rev. Lett. 33, 1139 (1974).

5. J.B. Taylor, Relaxation and magnetic reconnection in plasmas, Rev. Mod. Phys. 58, 741 (1986).

6. S.C. Hsu and P.M. Bellan, Experimental identification of the kink instability as a poloidal flux amplification mechanism for coaxial gun spheromak formation, Phys. Rev. Lett. 90, 215002 (2003).

7. Furno et al., Reconnection scaling experiment: a new device for three-dimensional magnetic reconnection studies, Rev. Sci. Instrum. 74, 2324 (2003).

\section{Role of small toroidal flux in FRCs}

The general sentiment among the participants was that the influence of toroidal fields on FRC behavior should have a high priority in FRC research, in theory, numerical simulations, and experiments.

\subsection{Ideal FRC}

In its "idealized" form, the field-reversed configuration (FRC) is assumed to have no regular toroidal field. An equilibrium is entirely determined by the poloidal field with field nulls situated in the O-point and in (usually two) X-points. As has recently been shown [Ryutov2004], the magnetic structure in the "poloidal field only" configurations is very sensitive to possible non-axisymmetric perturbations of the magnetic field. Perturbations with low mode numbers, even if their amplitude is very small, cause large radial wondering of the field lines, 
comparable to the separatrix radius. This may lead to an increase of transport, especially electron heat transport. The magnitude of this increase depends on the electron collisionality. In high-density MTF experiments as well as other present-day experiments in which the plasma collisionality is high because of relatively low temperature, the effect is smaller than in lowcollisionality systems, such as steady-state FRCs.

\subsection{Transport}

For non-axisymmetric perturbations introduced by external sources (e.g., RF perturbations intended for the current drive), one can substantially reduce radial transport by maintaining a proper parity of perturbations [Cohen2000]. The analysis of the electron heat transport in FRCs was presented at the Workshop in paper [Ryutov2004_CT2004]. It was shown that substantial reduction of the electron heat transport can be reached by introducing a regular toroidal magnetic field that exceeds by a factor of a few the amplitude of the magnetic field perturbations. For example, for perturbations of a relative amplitude of $3 \%(\sim 0.1 \%$ in terms of the magnetic energy), the toroidal magnetic field with an amplitude $\sim 10 \%$ of the poloidal field will already suppress the electron thermal conduction. Note that $10 \%$ toroidal field is too weak to cause any substantial changes in the plasma equilibrium; in other words, the FRC remains quite different from a spheromak, where the toroidal field is much larger, approximating force-free low- $\beta$ configuration. In order to have a substantial effect on the transport, the weak toroidal field must have an even parity with respect to the equatorial plane of FRC.

If a toroidal field is present, a new loss channel appears [Ryutov2004_CT2004]., which is somewhat reminiscent of a neoclassical transport in other toroidal devices. This time it acts mostly on the ions. In the strongly collisional case this transport mechanism becomes unimportant. In the case of a strongly elongated FRC (with $E=L / d ~>>1$ ) even a weak toroidal magnetic field, $B_{t} / B_{p} \sim 1 / E$, may have a strong effect on the gross stability. This happens because the $q$ value scales as $\mathrm{EB}_{\mathrm{t}} / \mathrm{B}_{\mathrm{p}}$ and may become of order 1 even at a weak toroidal magnetic field (provided it is an even function with respect to the equatorial plane). At the same time, the toroidal field has only a minor effect on the FRC equilibrium, $\sim 1 / \mathrm{E}^{2}<<1$. Very little (if anything) is known about the MHD stability in this setting. The issue deserves some attention of the theorists. In the discussions during the Workshop, it was noted that, in the situation where $\mathrm{q}$ becomes of order 1, the magnetic field perturbations lead to formation of "canonical" islands and may lead to confinement degradation if the islands are thick enough. Stochastization of the magnetic field by overlapping higher-order islands is also possible.

\subsection{Experimental and computational results}

On the experimental front, the spontaneous generation of weak toroidal fields is known to be a rather common occurrence. In Ref. [Tuszewski1989] the formation of a toroidal field with an even parity was reported. In Ref. [Guo2004], an interesting evolution of the toroidal magnetic field in the translation-reflection experiment was reported: initially, the plasmoid initially has little poloidal flux but strong toroidal flux at the ends with odd parity. In a numerical study [Omelchenko2000], formation of toroidal field with odd parity was reported. After superAlfvénic reflections from the end magnetic mirrors, the plasmoid settles down into a near-FRC state. Most of the oppositely directed toroidal flux was annihilated, with only a reduced unidirectional toroidal flux remaining (the parity becomes even). Substantial flux conversion from toroidal to poloidal occurs with approximate preservation of the magnetic helicity. This is a tribute not only to the robustness of FRCs, but to the tendency of an FRC to assume a preferred 
plasma state. The elongation at this stage is $\sim 10$, so that the observed toroidal field $\mathrm{Bt} \sim(1 / 5) \mathrm{Bp}$ can already create the situation with $\mathrm{q} \sim 1$ over most of the $\mathrm{FRC}$, with a strong shear near the $\mathrm{B}_{\text {pol }}$ $=0$ flux surface. The flux lifetime of these FRCs is significantly better than predicted by the conventional non-translated FRC scaling.

Further evidence comes from the Rotating Magnetic Field (RMF) formed and sustained FRCs. FRCs have been formed and sustained for up to 50 normal flux decay times by RMF in TCS [Hoffman2004]. During these longer pulse FRCs, a sudden transition to a higher performance mode occurs, accompanied by the spontaneous generation of toroidal fields. The toroidal fields are predominantly concentrated near the field null, with local q values significantly greater than unity. In MRX-FRC at Princeton [Ji2004], provisions are made to create FRCs with arbitrary toroidal flux. In the context of the aforementioned issues, this would be a very interesting experiment.

\subsection{Existence of intermediate states between FRC and spheromak}

It was noted during the meeting by several participants that it is unclear whether there exists a continuum of long-lived configurations spanning from the "idealized" FRC at one extreme (zero toroidal field, beta 1), and the "idealized" spheromak configuration at the other (comparable toroidal and poloidal fields, relatively low beta). The question was formulated: "Do there exist intermediate configurations with beta, say, equal to 0.3?" Experimental evidence to date casts doubt on this possibility. A range of compact toroids have been generated in the same facility (TS-3/4), but a clear gap is observed between FRC-like and spheromak-like states. Transitional states appear to in the theory of two-fluid minimum energy states [Steinhauer2002], but the transitional states may or may not be "attractors" in the nonlinear dynamics of the relaxation. Determining if there is a continuum between stable FRCs and spheromaks and possibly spherical tokamaks is an important step required to advance the understanding of generalized relaxation.

1. L.C. Steinhauer, Phys. Plasmas 9, 3767 (2002).

3. D.D. Ryutov, J. Kesner and M.E. Mauel. Phys. Plasmas, 11, 2318 (2004).

4. S.A. Cohen, R.D. Milroy. Phys. Plasmas, 7, 2539 (2000).

5. D.D. Ryutov. Enhanced transport caused by magnetic field perturbations in field reversed configurations. Paper presented at the US-Japan Compact Torus Workshop, Santa Fe (NM), September 14-16, 2004.

6. M. Tuszewski and B.L. Wright. Phys. Rev. Lett, 63, 2236 (1989).

7. H.Y. Guo, A.L. Hoffman, K.E. Miller, L.C. Steinhauer. Phys. Rev. Lett., 92, 245001-1 (2004)

8. Yu. A. Omelchenko. Phys. Plasmas, 7, 1443 (2000).

9. A.L. Hoffman, H.Y. Guo, K.E. Miller and R.D. Milroy, K.E. Miller, Long Pulse FRC Sustainment with Enhanced Edge Driven Rotating Magnetic Field Current Drive, to be presented at 20th IAEA Fusion Energy Conference, November 1-6, 2004, Vilamoura, Portugal.

10. H. Ji, M. Yamada, S. Gerhardt, E. Belova, D. Mikkelsen, S. Zweben, R. Davidson and R. Kaita. Sustainment of stable Field-Reversed Configuration by Neutral Beam Injection and Current Transformer. Paper presented at the US-Japan Compact Torus Workshop, Santa Fe (NM), September 14-16, 2004.

\section{Flow measurements}

\subsection{Stabilizing effect of flow}

The importance of equilibrium flows in plasmas is now broadly recognized. It has appeared in contexts as widely ranging as the L-to-H transition in tokamaks to the rotational instability in FRCs. Rotational flow in FRCs has long been known and occasionally measured because of its destabilizing effect. However, the possible stabilizing effect of flows, especially sheared flows, 
has only recently emerged as a result of studies of two-fluid minimum-energy states. Poloidal flows may impart a rigidity to the plasma, which combined with rotational (toroidal) flow, may give gyroscopic stabilization [Geren2004].

1. P. Geren and L.C. Steinhauer, Phys. Plasmas 11, 3646 (2004).

Flow measurements.

Methods for measuring flows are not nearly as mature as those for magnetic fields. Yet, the flow field, both poloidal and toroidal, must be known in order to track the evolution of the ion helicity, and to understand instabilities (e.g., kink modes) that serve to reorganize the plasma and possibly spawn further self-generated flows. The physical mechanisms for these transitions, including two-fluid and gyroviscous effects, also need to be illuminated. For instance, we know almost nothing about the magnitude of plasma viscosity. A concerted laboratory based effort to realize successful techniques to measure flow would have a large scientific payoff in the ICC and CT communities. Technologies to drive flow, such as biased electrodes and plasma gun methods, will also be useful not only to take advantage of different equilibria that may be accessible but also to investigate the physics systematically.

\subsection{Angular momentum.}

The influence of angular momentum conservation needs further study. This includes determining what boundary conditions either promote or compromise its conservation. If angular momentum is essential for stability, then practical methods for assuring adequate rotation should be explored.

\section{Importance of current drive, flux maintenance}

\subsection{Current Drive}

Current drive is a key concern for all current-carrying toroidal devices. This process is somewhat unique in CTs as there is no Ohmic transformer. Since the boundaries are less established than in toroidal devices, it is usually more appropriate to talk about poloidal flux maintenance. The power required will depend on the resistivity of the plasma. In FRCs the toroidal current is all diamagnetic, so the resistivity will be anomalous. However, since beta is high the required magnetic field strengths will be relatively low, and the current densities need not be too high. For spheromaks, RFPs, and to some extent STs, the currents that must be maintained are quite large.

\subsection{Flux Maintenance in FRCs}

The only two methods proposed for sustaining FRCs are RMF and Tangential Neutral Beam Injection (TNBI). RMF generally drives current near the separatrix, while TNBI is most efficient near the field null. The two methods should probably be used together since they supply momentum in opposite directions and could conceivably be used to influence both current profiles and toroidal velocity shear. The physics of RMF drive has been fairly well developed. On the other hand, while neutral beams have been injected into FRCs, the FRC flux levels have been too low to trap the resultant high energy ions inside the separatrix. Thus, the utility of TNBI for FRC current maintenance has not yet been tested.

\subsection{Flux Maintenance in Spheromaks and RFPs}

Helicity injection has been considered as a method for maintaining flux and current in both spheromaks and RFPs. In spheromaks toroidal flux is supplied by a coaxial gun injecting poloidal current at the edge, and a dynamo process maintains toroidal current in the center. In 
RFPs, poloidal flux is supplied through an Ohmic transformer, but this is not a true steady state method, and current maintenance for longer than the transformer flux swing time may be very difficult. In either case, reliance on the relaxation principal to provide the desired flux ratios may result in turbulence which unfavorably affects plasma confinement.

\section{CT Power-Plant Considerations}

Only a modest amount of recent work has been performed on CT-related fusion power-plant topics. There remains a general appreciation of the flexibility of the CT class in addressing the perceived needs and opportunities of future power stations. A detailed assessment of the competitive position of CTs relative to mainline MFE and IFE candidates must await further development of the various CT approaches. The potential for achieving high values of plasma beta in some CTs allows anticipation of advanced-fusion-fuel (e.g., D-3He) applications with high-efficiency direct conversion of charged-particle energy. Such systems result in greatly reduced neutron production and associated neutron damage of structural materials, with favorable consequences for the power-plant operational availability and radioactive-waste disposal. For D-T fueled systems, high plasma beta may result in high power density. On the positive side, a high-power-density fusion system may reap direct-cost savings. Adverse consequences include possible high $14-\mathrm{MeV}$ neutron wall loads coupled to fluence-limited structural lifetimes and high surface heat fluxes.

Fortunately, the geometries of CT systems may more easily accommodate certain mitigating technologies (e.g., liquid walls) than can tokamaks and stellarators. Operationally, CT systems range from steady-state to pulsed. The ability to translate certain CTs allows physical separation of the plasma formation apparatus from the burn chamber. Adiabatic compression (possibly liner driven) may allow heating to ignition. Various combinations of these features have resulted in a number of power-plant embodiments over the years.

Pulsed systems require extra fusion gain [Q fusion yield divided by invested (plasma) energy] to compensate for the dwell time between pulses. High yields require clever stand-off schemes and/or cheap and rapid remanufacture of destroyed components and may be accomplished in IFE-type chambers protected by thick-liquid falls.

\section{Summary}

This CT workshop provided an opportunity for a small subset (25-30 people) of the ICC community to get together and discuss issues that are directly related to CT physics. Many of our fusion reactor scenarios are intrinsically pulsed or cyclic, operate at large beta, and have not received the attention or support enjoyed by the proponents of a steady state path. The physics of relaxation and equilibrium at large beta still needs much further study. Moreover, there is a strong connection between the fundamental science that is crucial to all the CT concepts and many interesting questions in space physics and astrophysics. We hope that this workshop continues in future years. Information is posted at our website http://wsx.lanl.gov/CT_workshop_2004

This work was supported by United States Department of Energy, Office of Science and Technology, Contract W-7405-ENG-36

This work was performed under the auspices of the U.S. Department of Energy by University of California, Lawrence Livermore National Laboratory under contract W-7405-Eng-48. 


\section{Addendum. List of participants}

$\begin{array}{llll}\text { Bellan } & \text { Paul } & \text { Caltech } & \text { USA } \\ \text { Dorf } & \text { Leonid } & \text { LANL } & \text { USA } \\ \text { Fujino } & \text { T } & \text { Nihon Univ } & \text { Japan } \\ \text { Furno } & \text { Ivo } & \text { LANL } & \text { USA } \\ \text { Geren } & \text { Preston } & \text { U Wash } & \text { USA } \\ \text { Gerhardt } & \text { Stefan } & \text { PPPL } & \text { USA } \\ \text { Gota } & \text { Hiroshi } & \text { Nihon Univ } & \text { Japan } \\ \text { Guo } & \text { Houyang } & \text { U Wash } & \text { USA } \\ \text { Hoffman } & \text { Alan L. } & \text { U Wash } & \text { USA } \\ \text { Hsu } & \text { Scott } & \text { LANL } & \text { USA } \\ \text { Intrator } & \text { Thomas } & \text { LANL } & \text { USA } \\ \text { Ji } & \text { Hantao } & \text { PPPL } & \text { USA } \\ \text { Kanki } & \text { T } & \text { Coast Guard Acad } & \text { Japan } \\ \text { Kawamori } & \text { E } & \text { Univ Tokyo } & \text { Japan } \\ \text { Milroy } & \text { Richard D. } & \text { U Wash } & \text { USA } \\ \text { Nagata } & \text { Masayoshi } & \text { Univ Hyogo } & \text { Japan } \\ \text { Okada } & \text { Shigefumi } & \text { Osaka Univ } & \text { Japan } \\ \text { Renneke } & \text { Richard } & \text { LANL } & \text { USA } \\ \text { Ruden } & \text { Edward L. } & \text { AFRL } & \text { USA } \\ \text { Ryutov } & \text { Dmitri } & \text { LLNL } & \text { USA } \\ \text { Sieck } & \text { Paul } & \text { U Wash } & \text { USA } \\ \text { Slough } & \text { John } & \text { U Wash } & \text { USA } \\ \text { Steinhauer } & \text { Loren } & \text { U Wash } & \text { USA } \\ \text { Takahashi } & \text { Toshiki } & \text { Gunma Univ } & \text { Japan } \\ \text { Takahashi } & \text { Tsutomu } & \text { Nihon Univ } & \text { Japan } \\ \text { Tang } & \text { Xianzhu } & \text { LANL } & \text { USA } \\ \text { Zhang } & \text { Shouyin } & \text { LANL } & \text { USA } \\ & & & \end{array}$

